\title{
Neutrino masses, states and interactions
}

\section{Susanne Mertens}

Max Planck Institute for Physics, Foehringer Ring 6, 80805 Munich, Germany

Technical University Munich, James Frank Str., 85748 Garching, Germany

E-mail: merten. ampp.mpg . de

\section{Stefano Morisi}

INFN, Sezione di Napoli, Complesso Univ. Monte S. Angelo, I-80126 Napoli, Italy

Dipartimento di Fisica Ettore Pancini, Universita di Napoli Federico II

E-mail: stefano.morisi@gmail.com

This paper summarizes the parallel session IV: "Neutrino masses, states and interactions". This session covered experimental and theoretical aspects of the absolute neutrino mass scale, searches for neutrinoless double beta decay, and searches for sterile neutrinos in different mass regimes. Beyond that, lepton flavor violation in the charged lepton sector, exotic interactions in tritium beta decay, and electromagnetic properties of neutrinos were discussed.

Neutrino Oscillation Workshop

4 - 11 September, 2016

Otranto (Lecce, Italy) 


\section{Introduction}

The origin and the nature of neutrino is still a mystery and is one of the biggest question in physics, theoretically and experimentally. In both contexts it is fundamental to know how neutrinos get mass and if neutrinos are Majorana or Dirac particles. The neutrino mass mechanisms and their nature are intimately related and any experimental progress on the determination on neutrino's nature will improve definitively also the knowledge about the neutrino mass mechanism. On the other hand knowing the nature of neutrinos will not fix completely the problem of the mass generation. In order to better understand this important properties it is required to learn also about neutrino interactions. This is the reason of the title of this IV parallel section.

In the parallel session "Neutrino masses, states and interactions" eight experimental and five theoretical talks were presented. The experimental talks comprised neutrinoless double beta decay searches, direct neutrino mass measurements, and searches for sterile neutrinos. The theory part was composed of two talks on the origin of neutrino mass, two talks on their interactions and one on the nature on neutrino.

\section{Theory}

In the standard model neutrinos are massless at the renormalizable level, but neutrinos get mass by means of the Weinberg non-renormalizable operator [1]

$$
\mathscr{L} \supset \frac{y_{i j}^{v}}{\Lambda} L_{i} \tilde{H} L_{j} \tilde{H}
$$

where $\Lambda$ is an effective mass scale and $y_{i j}^{v}$ is an arbitrary matrix. Such an operator gives rise to a Majorana mass for neutrinos. However we do not know if neutrinos are Dirac or Majorana particles and so we do not know if such an effective operator is present or not. If present, it is important to understand the renormalizable theory that is hidden in the Weinberg operator. At the renormalizable level there are many possibilities at three level as well as from radiative contributions and in both scenarios we can have new physics at high scale (of the order of the grand unified scale) or at low scale. At tree level we can have the standard seesaw mechanisms denoted as type-I, II or III (see for instance [2]) depending if the mediator is a fermion $\mathrm{SU}(2)$ singlet, scalar $\mathrm{SU}(2)$ triplet or fermion SU(2) triplet, respectively. The mediators can have very different mass scales but typically high-mass scales are quite natural for such kind of mechanisms. Lopez-Pavon has presented a counterexample where the right-handed neutrino can be just above the electroweak scale in a typeI seesaw mechanism but the Dirac Yukawa couplings have not to be small giving rise to interesting non-unitarity effects that can be tested in lepton flavor violation experiments. On the other hand there is a class of mechanisms (inverse and linear seesaw) where the new physics scale can be naturally low, around $\mathrm{TeV}$ (see [3] for a review). But the Weinberg operator can be realized at the renormalizable level also radiatively by means of one-loop diagrams like the Zee model [4], two-loop Feynman diagram Zee-Babu model [5, 6] and even at three loops [7].

All these extensions of the standard model are possible realizations of the Weinberg operator and give rise to a Majorana mass for neutrino. On the other hand if neutrino are pure Dirac particles we have to extend the Standard Model including the right-handed neutrino fields. So Majorana/Dirac makes a big difference from the theoretical point of view In such a case it is quite 
difficult to explain the suppression of the neutrino masses compared to the other charged Dirac fermions. Unfortunately we have no way to prove experimentally if neutrino are Dirac particles, but if neutrino have Majorana nature, then they predict the so called neutrinoless double beta decay $(0 v \beta \beta)$ with a violation of the lepton number of two units $\Delta L=2$. It is alo true the opposite, namely if $0 v \beta \beta$ will be observed then neutrino are Majorana particles (black box theorem [8]). The $0 v \beta \beta$ is proportional to a parameter related to the neutrino physics, that is the ee element of the neutrino mass matrix $\left|\left(m_{v}\right)_{e e}\right|=\left|\sum_{i} U_{i e} m_{i}\right|$ where $U$ is the neutrino mixing matrix and $m_{i}$ the physical mass neutrino masses. The measured $0 v \beta \beta$ half-life time is proportional to the parameter $\left|\left(m_{v}\right)_{e e}\right|$. On the other hand in order to extract such a parameter from $0 v \beta \beta$ half-life it is crucial the precise determination of the nuclear matrix elements. This is an important and complicate experimental and theoretical task and has been summarized by Simkovic.

As the KATRIN experiment will soon start data taking, it is interesting to investigate nonstandard decay modes of tritium. Especially, with the possible realization of TRISTAN, which targets a measurement of the entire tritium spectrum, exotic interactions leading to a deformation anywhere in the spectrum can be investigated. In the talk by Patrick Ludl on "Exotic charged current interactions in tritium beta decay" all possible Lorentz-invariant interactions were investigated in an effective operator approach [9]. In particular the case of $\mathrm{keV}$-scale sterile neutrinos and right-handed currents was discussd. Interestingly, keV-sterile neutrino models can lead to sizable signatures in the beta decay spectrum, while not being in contradiction to X-ray limits or overclosure bounds [10].

On the top of such a complicated puzzle, there is another problem that is deeply connected with the fermion masses generation: the flavor problem. We know that quarks of different flavors are mixed as well as neutrinos. On the other hand the mixings are very different in the quark and lepton sectors. Moreover fermion masses show a extremely wide range that span 12 order of magnitude. It could be that such a patterns hide some dynamical mechanism or some underlying symmetry. There are in first approximation three classes of possibilities, by extending the Standard Model with gauge vertical symmetry, like in the case of Grand Unified Theories (GUT) or with horizontal families symmetries, or combination of vertical and horizontal symmetries. This is the case of the talk presented by D. Meloni on "(not only) neutrino and GUTs". It has been showed that starting form a flavor symmetry in GUT it yields a quark-lepton complementary mixing relation, $\theta_{12}+\theta_{C} \approx \pi / 4$ where $\theta_{12}$ is the lepton mixing angle and $\theta_{C}$ is the quark Cabibbo angle (that is almost true experimentally). The family symmetry used is $S_{4}$ the permutation of four objects, and the grand unified group considered are $\mathrm{SU}(5)$ and $\mathrm{SO}(10)$.

An example of neutrino interaction has be discussed by Studenikin that in particular has given a report on the status of the electromagnetic interaction of neutrino.

\section{Experiments}

The experimental part of the session covered lepton number violaton in the charged lepton sector with the MEG experiment, neutrino mass measurements with the KATRIN experiment, new ideas for laboratory searches for keV-scale sterile neutrinos, a variety of experiments searching for neutrinoless double beta decay, and finally the NUMEN experiment, which is aiming to determine the relevant nuclear matrix elements for neutrinoless double beta decay. 
MEG Experiment The Mu-to-E-Gamma (MEG) experiment searches for charged lepton number violation (cLFV) with muons. Lepton number is conserved in the Standard Model (SM), but is naturally violated in Physics beyond the SM. Hence, searches for rare or SM-forbidden decays, allow to reveal or constrain new physics contributions.

The MEG experiment uses an intense DC muon beam of $10^{8}$ muons/s at low momentum of $29 \mathrm{MeV} / \mathrm{c}$, provided by the piE5 beam line at PSI. The signature of $\mu^{+} \rightarrow e^{+}+\gamma$ is a coincidence signal of the back-to-back emitted positron and mono-energetic gamma. A liquid xenon scintillator measures the gamma energy and a drift chamber in a magnetic field records the track of the positrons.

With the full data set accumulated from 2009 to 2013, the MEG collaboration published the world's leading upper limit of the branching ratio of $\mathscr{B}\left(\mu^{+} \rightarrow e^{+}+\gamma\right)<4.2 \cdot 10^{-13}$ [11]. MEG-II is designed to improve the sensitivity by one order of magnitude by 2020 .

The KATRIN Experiment The KATRIN experiment is designed to directly measure the effective electron anti neutrino mass via tritium $\beta$ decay. A non-zero neutrino mass reduces the endpoint $E_{0}$ of the $\beta$ decay and distorts the spectral shape close to $E_{0}$. KATRIN is combining a high-luminous gaseous molecular tritium source with a high-resolution spectrometer, operating as a MAC-E filter (Magnetic Adiabatic Collimation with Electrostatic filter) [12]. The design sensitivity is $200 \mathrm{meV}$ at $90 \% \mathrm{CL}$ after three years of measurement time.

Since 2016 all components of the KATRIN experiment, including rear-wall, tritium source, transport and pumping section, spectrometers, and focal plane detector, are on-site at the Karlsruhe Institute of Technology, Germany. As announced in the presentation, a major milestone, the "first light" of KATRIN, was achieved in October 2016: electrons, created at the rear wall of KATRIN, were transmitted through the 70-m-long setup and reached the focal plane detector. This demonstrates the functionality of the magnet system and good alignment of all components. First tritium measurements are planned for end of 2017.

Laboratory searches for $\mathbf{k e V}$ sterile neutrinos Right-handed (and hence sterile) neutrinos are a natural extension of the Standard Model. The scale of the corresponding new neutrino mass eigenstate, however, is completely unknown. $\mathrm{keV}$-scale sterile neutrinos are motivated as dark matter candidate [13].

Beyond measuring the neutrino mass, KATRIN - equipped with a new focal plane detectorhas the capability to search for $\mathrm{keV}$-scale sterile neutrinos. New keV-scale neutrino mass eigenstates would leave a kink-like signature in the tritium $\beta$-decay spectrum, at an energy $E=E_{0}-m_{s}$, where $m_{s}$ is the mass of the new neutrino eigenstate. The amplitude of the signature is determined by the active-sterile-mixing amplitude $\sin ^{2} \Theta$. In the context of the TRISTAN project of KATRIN a novel detector and read-out system is being developed that would allow to measure the entire tritium $\beta$-decay spectrum (not only the endpoint region) with a precision in the ppm-level. A multi-pixel Si-drift detector design is foreseen $[14,15]$

Another way to search for dark matter keV-scale sterile neutrinos is via their direct detection. Here, a completely new idea was presented, which exploits the inverse $\beta$ decay on the stable target ${ }^{137} \mathrm{Dy}+v_{s} \rightarrow{ }^{137} \mathrm{Ho}+e .{ }^{137} \mathrm{Dy}$ is "almost" $\beta$ decaying, and hence the mass of the keV-scale 
sterile neutrino is sufficient to make the inverse beta decay possible. First background studies, and different measurement modes have been proposed [16].

NEXT NEXT is an experiment to search for $0 v \beta \beta$-decay in ${ }^{137} \mathrm{Xe}$ with a high pressure Xenon (HPXe) Time Projection Chamber (TPC). ${ }^{137} \mathrm{Xe}$ has an endpoint of $Q_{\beta \beta}=2458 \mathrm{keV}$ and an isotopic abundance of $8.9 \%$. The electrons emitted in the decay produce a prompt scintillation signal, detected by TMPs, and an ionization (amplified via electroluminescence) signal, measured by SiPMs. The latter signal allows to reconstruct the electron track, which is essential to assess the event topology and hence discriminate signal from background.

Two prototypes, called NEXT-DEMO and NEXT-DBDM, were operated at IFIC, Valencia, Spain and Lawrence Berkeley National Laboratory (LBNL). They contained about $1 \mathrm{~kg}$ of natural $\mathrm{Xe}$ at $10^{-15}$ bar. Both systems could achieve an energy resolution of $0.5-0.6 \%$ FWHM at $Q_{\beta \beta}$ and demonstrated the characteristic event topology.

The next stage, NEXT-WHITE, scheduled from 2016 to 2019 is designed to measure the halflife of the two neutrino mode and to test the background model. The detector is fully commissioned at the Laboratorio Subterraneo de Canfranc (LSC) and first data is expected in 2017. With the subsequent NEXT-100 a background rate of $4 \cdot 10^{-4} \mathrm{c} / \mathrm{keV} / \mathrm{kg} / \mathrm{y}$ and a sensitivity of $T_{1 / 2} \approx 5 \cdot 10^{25} \mathrm{y}$ at $90 \% \mathrm{CL}$ is expected.

The CUORE and CUORE-0 experiments CUORE is an experiment to search for $0 v \beta \beta$ in ${ }^{130} \mathrm{Te} .{ }^{130} \mathrm{Te}$ has an endpoint of $Q_{\beta \beta}=2528 \mathrm{keV}$ and high natural abundance of $34.2 \%$. CUORE is using a bolometric techniques where the energy of the electrons is detected by a temperature increase of the ${ }^{\text {nat }} \mathrm{TeO}_{2}$ crystals. An excellent energy resolution of $5 \mathrm{keV}$ at $Q_{\beta \beta}$, but no particle identification can be achieved.

With CUORE- 0 , a single tower of 52 detectors, an exposure of $9.8 \mathrm{~kg} \cdot \mathrm{yr}{ }^{130} \mathrm{Te}$ was accumulated and a limit of $T_{1 / 2}>4.0 \cdot 10^{24} \mathrm{y}$ (combined with Cuoricino) was set [17]. In 2016, the full CUORE detector, consisting of 19 towers was completed. The background goal is $10^{-2} \mathrm{c} / \mathrm{keV} / \mathrm{kg} / \mathrm{y}$ and the expected sensitivity is $9.5 \cdot 10^{25} \mathrm{y}$ at the $90 \% \mathrm{CL}$ after 5 years of data taking.

Beyond CUORE several upgrade options are discussed in the context of CUPID. These entail enrichment of the tellurium, an extension of the detector technology to allow for a particle ID, and the usage of a different isotope.

GERDA GERDA is searching for $0 v \beta \beta$ in ${ }^{76} \mathrm{Ge} .{ }^{76} \mathrm{Ge}$ has an endpoint of $Q_{\beta \beta}=2039 \mathrm{keV}$ and a natural abundance of $7.6 \%$. GERDA is using bare, high-purity germanium detectors enriched to $86 \%$ in ${ }^{76} \mathrm{Ge}$. The detector array is immersed in liquid argon (LAr) as cooling and active veto. GERDA is operating so-called Coaxial (coax) detectors and Broad Energy Germanium (BEGe) detectors, providing an excellent energy resolution of 3-4 keV FWHM at $Q_{\beta \beta}$. The BEGe detectors allow for an efficient background discrimination based on the signal pulse shape.

In GERDA Phase-1 $17.7 \mathrm{~kg}$ of coaxial detectors and 5 BEGe detectors were installed. With a total exposure of $21.6 \mathrm{~kg} \cdot \mathrm{yr}$ a limit of $T_{1 / 2}>2.1 \cdot 10^{25} \mathrm{y}$ was achieved [18]. In GERDA PhaseII 30 additional BEGe's were added to the detector array and the LAr was used as active veto. Additionally, the individual detector strings were surrounded by a nylon shroud to prevent ${ }^{42} \mathrm{~K}$ from reaching the detectors. The commissioning was completed in December 2015. For the first data release an exposure of $5.8 \mathrm{~kg} \cdot \mathrm{yr}$ for enriched BEGe and $5.0 \mathrm{~kg} \cdot \mathrm{yr}$ for enriched coax was 
collected. An unprecedented background level of $0.7 \cdot 10^{-3} \mathrm{c} / \mathrm{keV} / \mathrm{kg} / \mathrm{y}$ was achieved with the BEGe detectors. A half-life limit of $T_{1 / 2}>5.2 \cdot 10^{25} \mathrm{y}$ at $90 \% \mathrm{CL}$ could be set. With the full exposure of $100 \mathrm{~kg} \cdot \mathrm{yr}$ a limit of $T_{1 / 2}>1 \cdot 10^{26} \mathrm{y}$ is expected.

AMoRE AMoRE is searching for $0 v \beta \beta$ in ${ }^{100} \mathrm{Mo}$, which has with $Q_{\beta \beta}=3034 \mathrm{keV}$ an endpoint, that lies above all natural-radioactivity-induced backgrounds. The isotopic abundance of ${ }^{100} \mathrm{Mo}$ is 9.6\%. AMoRE is using the ${ }^{40} \mathrm{Ca}^{100} \mathrm{MoO}_{4}$ crystals combined with a Ge-wafer to detect both scintillation light and heat. Metallic Magnetic Calorimeters (MMC) are used both to detect the scintillation-photon-induced heat in the Ge-wafer and the heat increase of the crystal itself.

An AMoRE Pilot ran underground with 5 crystals of $1.5 \mathrm{~kg}$ total mass. An energy resolution of approximately $20 \mathrm{keV}$ at $Q_{\beta \beta}$ was demonstrated. A phased-approach is planned with AMoRE-I, consisting of $5 \mathrm{~kg}$ of crystals to be operated from 2017 to 2019, followed by AMoRE-II, consisting of $200 \mathrm{~kg}$ of crystals, to be operated from 2019 to 2024. The background goal of AMoRE-II is $4 \cdot 10^{-4} \mathrm{c} / \mathrm{keV} / \mathrm{kg} / \mathrm{y}$ and the expected sensitivity is $T_{1 / 2}>5 \cdot 10^{25} \mathrm{y}$.

NUMEN The aim of the NUMEN Project is to access the nuclear matrix elements (NMEs) relevant for $0 v \beta \beta$. The idea is to measure the cross sections of Heavy-Ion-induced (HI) DoubleCharge-Exchange (DCE) reactions with high accuracy. This reaction has the same initial and final states as $0 v \beta \beta$ and very similar transition operators. The description of NMEs extracted from DCE and $0 v \beta \beta$ decay is similarity complex, however DCE has the advantage to be accessible in laboratory.

Currently, the DCE process ${ }^{40} \mathrm{Ca}\left({ }^{18} \mathrm{O},{ }^{18} \mathrm{Ne}\right){ }^{40} \mathrm{Ar}$ at $270 \mathrm{MeV}$ incident energy is investigated at INFN-Laboratori Nazionali del Sud Catania, Italy. A superconducting cyclotron is used to accelerate the ions and a MAGNEX spectrometer is used to detect and measure the energy of the reaction products. The experiment could demonstrate that the cross section factorization in a nuclear structure term containing the matrix elements and a nuclear reaction factor reasonably holds for the relevant transitions [19]. To access the interesting cases for $0 v \beta \beta$, higher beam currents, gaseous targets, and better energy resolution will be needed. Substantial upgrades of the accelerator and the detector are planned. First measurements with an upgraded system and a few selected isotope candidates are planned for 2016 - 2018. The final measurements at high beam intensity and for all isotopes of interest are planned to start in 2021, after a major facility upgrade.

\section{References}

[1] S. Weinberg, Phys. Rev. Lett. 43, 1566 (1979). doi:10.1103/PhysRevLett.43.1566.

[2] W. Grimus, PoS P 2GC, 001 (2006) [hep-ph/0612311].

[3] S. M. Boucenna, S. Morisi and J. W. F. Valle, Adv. High Energy Phys. 2014, 831598 (2014) doi:10.1155/2014/831598 [arXiv:1404.3751 [hep-ph]].

[4] A. Zee, Phys. Lett. 93B, 389 (1980) Erratum: [Phys. Lett. 95B, 461 (1980)] doi:10.1016/0370-2693(80)90349-4, 10.1016/0370-2693(80)90193-8.

[5] A. Zee, Phys. Lett. 161B, 141 (1985). doi:10.1016/0370-2693(85)90625-2.

[6] K. S. Babu, Phys. Lett. B 203, 132 (1988). doi:10.1016/0370-2693(88)91584-5. 
[7] L. M. Krauss, S. Nasri and M. Trodden, Phys. Rev. D 67, 085002 (2003) doi:10.1103/PhysRevD.67.085002 [hep-ph/0210389].

[8] J. Schechter and J. W. F. Valle, Phys. Rev. D 25, 2951 (1982). doi:10.1103/PhysRevD.25.2951.

[9] Ludl, P.O. and Rodejohann, W. J. High Energ. Phys. (2016) 2016: 40. doi:10.1007/JHEP06(2016)040.

[10] Barry, J., Heeck, J. and Rodejohann, W. J. High Energ. Phys. (2014) 2014: 81. doi:10.1007/JHEP07(2014)081.

[11] Baldini, A.M., Bao, Y., Baracchini, E. et al. Eur. Phys. J. C (2016) 76: 434. doi:10.1140/epjc/s10052-016-4271-x.

[12] G. Drexlin, V. Hannen, S. Mertens, and C. Weinheimer, Advances in High Energy Physics, Volume 2013, Article ID 293986 (2013).

[13] R. Adhikari et. al. arXiv:1602.04816 [hep-ph].

[14] S. Mertens et al 2015 JCAP 150202020.

[15] S. Mertens et al 2015 Phys. Rev. D 914042005.

[16] T. Lasserre, K. Altenmueller, M. Cribier, A. Merle, S. Mertens, M. Vivier arXiv:1609.04671 [hep-ex].

[17] K. Alfonso et al. (CUORE Collaboration) Phys. Rev. Lett. 115, 102502.

[18] M. Agostini et al. (GERDA Collaboration) Phys. Rev. Lett. 111, 122503.

[19] Cappuzzello, F., Cavallaro, M., Agodi, C. et al. Eur. Phys. J. A (2015) 51: 145. doi:10.1140/epja/i2015-15145-5. 